\title{
Controllable deformation of silicon nanowires with strain up to $24 \%$
}

\author{
Sameer S. Walavalkar, ${ }^{\text {a) }}$ Andrew P. Homyk, M. David Henry, and Axel Scherer \\ Department of Applied Physics, Kavli Nanoscience Institute, Caltech, 1200 E. California Blvd, Pasadena, \\ California 91125, USA
}

(Received 16 February 2010; accepted 26 April 2010; published online 22 June 2010)

\begin{abstract}
Fabricated silicon nanostructures demonstrate mechanical properties unlike their macroscopic counterparts. Here we use a force mediating polymer to controllably and reversibly deform silicon nanowires. This technique is demonstrated on multiple nanowire configurations, which undergo deformation without noticeable macroscopic damage after the polymer is removed. Calculations estimate a maximum of nearly $24 \%$ strain induced in $30 \mathrm{~nm}$ diameter pillars. The use of an electron activated polymer allows retention of the strained configuration without any external input. As a further illustration of this technique, we demonstrate nanoscale tweezing by capturing $300 \mathrm{~nm}$ alumina beads using circular arrays of these silicon nanowires. (c) 2010 American Institute of Physics. [doi:10.1063/1.3436589]
\end{abstract}

\section{INTRODUCTION}

Designing, etching, and manipulating silicon nanowires has become increasingly important to a variety of research areas including nanoelectromechanical systems, optics/plasmonics, ${ }^{1}$ and nanoscale circuit elements. Specific applications include high surface area chemical sensors, ${ }^{2}$ mechanical oscillators, ${ }^{3,4}$ and piezoresistive sensors. High aspect ratio pillars with diameters between 50-100 nm could prove useful for core-shell type plasmonic resonators, ${ }^{5}$ while pillars with sub-10 $\mathrm{nm}$ diameters have shown promising light emission characteristics. ${ }^{6,7}$ High aspect ratio structures also have possible applications to high density electronics such as FinFETS (Ref. 3) or in DRAM devices.

Current fabrication and manipulation techniques suffer from a few limitations. For applications requiring precision placement of pillars, including electronics and optical coupling, a top-down fabrication scheme is required. However, even with current electron beam technology, alignment with accuracy greater than $10 \mathrm{~nm}$ is difficult. Mechanical manipulation using electrostatic actuation ${ }^{8,9}$ requires power to be continuously supplied to the device to maintain deformation. Other static deformation methods-such as pseudomorphic growth-lack the ability to reversibly tune the amount of strain in the pillars once they have been patterned, and cannot achieve strain $>2-3 \%$. ${ }^{10,11}$ Given its influence on electronic as well as optical properties, ${ }^{11-13}$ methods to accurately control strain are becoming increasingly important in modern devices.

Previous work has shown that nanoscale structures experience deformation differently than their bulk counterparts. ${ }^{14-19}$ Specifically silicon nanowires can demonstrate yeild strength as well as total strain much greater than bulk silicon. ${ }^{20,21}$ In two notable studies ${ }^{21,22}$ grown silicon nanowires were shown to have an elongation ratio of $125 \%$ and a maximum strain of $21.5 \%$ without failure. Furthermore previous efforts have utilized the length contraction of polymerization to apply significant forces to induce elongation,

${ }^{\text {a)} E l e c t r o n i c ~ m a i l: ~ w a l a v a l k @ c a l t e c h . e d u . ~}$ buckling, and bending of silicon nanowires and carbon nanotubes. $^{21-23}$ However, common limitations shared by these approaches are the use of grown nanowires or nanotubes resulting in stochastically distributed structures ${ }^{21,23}$ as well as the permanence of the deformed position achieved by the nanowire or nanotube.

Here we present a novel method of passively manipulating etched silicon nanowires using polymethyl-methacrylate (PMMA) as a force mediating polymer. The force exerted by the PMMA on the nanowires may be tuned by varying the electron beam exposure, heating, and selective polymer removal, permitting controllable, as well as fully reversible, bending and straining of structures. Due to the stability of PMMA at room temperature, the nanowire configuration remains fixed, enabling further electrical or mechanical tests to be performed on the sample while under strain. This paper details the nanowire fabrication, polymer manipulation, deformation characteristics, and sample applications of this technique.

\section{METHODS}

The fabrication of the nanowires follows Henry et al. ${ }^{24}$ pillars were defined by e-beam patterning an array of 30-150 $\mathrm{nm}$ disks in $75 \mathrm{~nm}$ of Micro-Chem PMMA 950 A2. A $25 \mathrm{~nm}$ layer of $\mathrm{Al}_{2} \mathrm{O}_{3}$ was deposited as a hard-mask via dc magnetron sputtering of aluminum with a 5:1 $\mathrm{Ar}: \mathrm{O}_{2}$ process chemistry and patterned via lift-off. Aluminum oxide has been demonstrated as a resilient as well as chemically inert etch mask $^{24}$ providing a selectivity of greater than 60:1 for a fluorine etch chemistry. Etching was performed in an Oxford Plasmalab 100 ICP-RIE 380 machine running a "Pseudo Bosch" etch with simultaneous etching using $\mathrm{SF}_{6}$ and passivation using $\mathrm{C}_{4} \mathrm{~F}_{8}$-so-called mixed-mode etching. Sidewall profiles are controlled by adjusting the ratio of etch to passivation gases. If diameter reduction was required, pillars were thermally oxidized down to a desired width and then dipped in buffered hydrofluoric acid to remove the oxide. An example of a fabricated nanowire array is given in Fig. 1. 


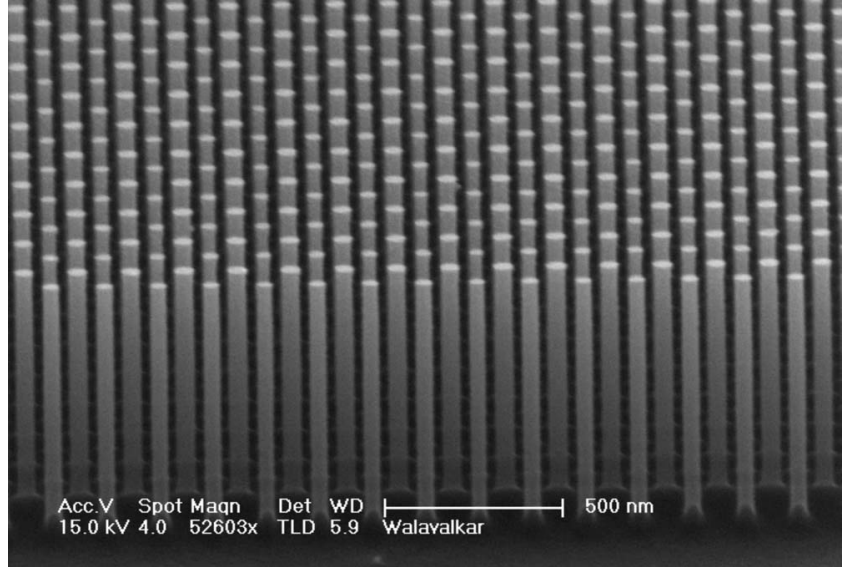

FIG. 1. An array of pillars fabricated via conventional top down CMOS processing. The pillars are $50 \mathrm{~nm}$ wide and 1.3 microns tall. The image is taken at $30^{\circ}$.

To prepare the sample for actuation, PMMA is spun over the pillar area and baked to drive off solvents. The PMMA is then planarized using an oxygen plasma to achieve the desired resist height.

It has been noted ${ }^{25,26}$ that under high electron beam doses-approximately ten times the standard dose used in electron beam lithography - the normally positive-tone PMMA crosslinks and behaves as a negative resist. Along with this change in resist tone, the crosslinking causes a volumetric contraction of the resist. We utilize this contraction to selectively deform pillars; directional control is achieved using asymmetric electron beam exposure.

Figure 2 shows two sets of $75 \mathrm{~nm}$ diameter nanowires emerging from a $300 \mathrm{~nm}$ thick PMMA film. By exposing only the right set of pillars, crosslinking in this area pulls these pillars inwards while neighboring strips remain undeformed. The inset shows the comparison in more detail. We note that the bottom few pillars of both the right and left patterns have been brought together from the exposure of taking this picture. The length of exposure dictates the electron dose and hence the amount of contraction between pillars. Since this exposure is done in an Scanning Electron

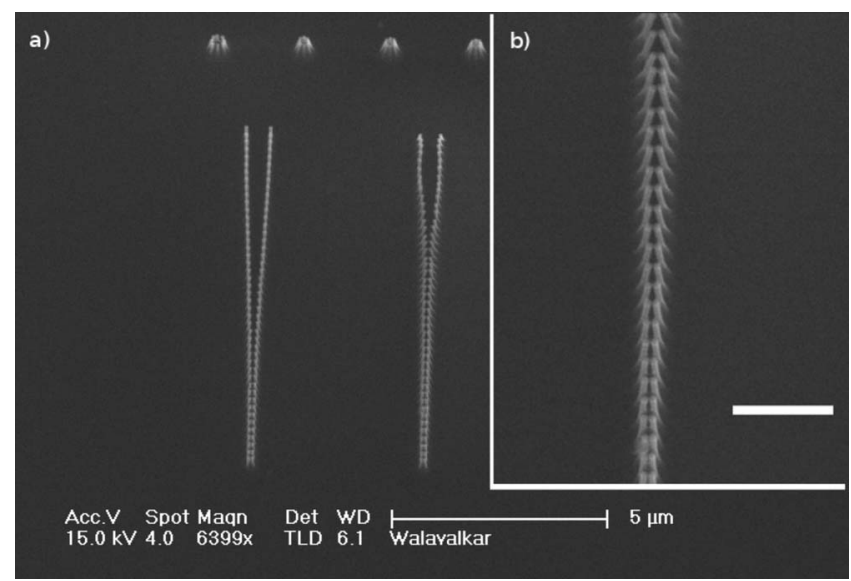

FIG. 2. (a) SEM of deformed strip next to an undeformed strip. (b) Closeup of lower section of deformed strip of pillars. Note the increased bend angle for the pillars that were originally further apart. Scale bar in inset is one micron.

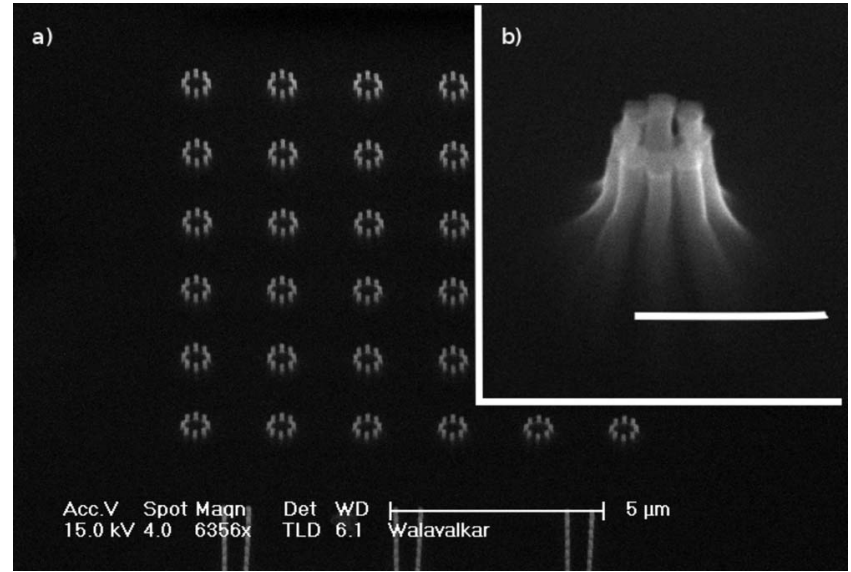

FIG. 3. (a) Array of $75 \mathrm{~nm}$ pillars in circular patterns with $500 \mathrm{~nm}$ diameter spacing. (b) Contraction of pillars after the exposure of the central region. Scale bar is $500 \mathrm{~nm}$.

Microscope (SEM), we could view the progress of the deformation in real time, freeze exposures, take measurements, and continue until a desired exposure was obtained. Using this process, it should be possible to obtain SEM resolution limited precision in the final positioning of the pillars.

In a second experiment, we arranged rings of eight nanowires $75 \mathrm{~nm}$ in diameter and $800 \mathrm{~nm}$ tall (Fig. 3). The rings were initially $500 \mathrm{~nm}$ in diameter with $230 \mathrm{~nm}$ circumferential spacing between the pillars. The central region of the ring was exposed in an SEM, yielding a uniform radial deformation. Contraction terminated when the pillars were flush, with a final diameter of $184 \mathrm{~nm}$ after exposure. As shown in Fig. 4, we also exposed the inner region of a ring of $50 \mathrm{~nm}$ diameter pillars that were $1 \mu \mathrm{m}$ tall. The ring started at $500 \mathrm{~nm}$ in diameter and contracted until the pillars were fully touching, giving a deformation distance at the tip of approximately $250 \mathrm{~nm}$. By measuring the beam current and exposure time, we determined that a dose of approximately $10000 \mu \mathrm{C} / \mathrm{cm}^{2}$ is required to achieve full contraction of the pillars. This dose matches other results for that required to fully-crosslink PMMA. ${ }^{26}$

An interesting consequence of overexposing the resist is its resilience to dissolution by acetone. ${ }^{25,26}$ Once the area of interest had been exposed, we dipped the sample in acetone to remove the nonoverexposed portions of resist, leaving the intended pillars locked in place while freeing the rest of the chip from PMMA. This permits further processing steps to be performed on the chip. Pillars can be relaxed by heating the chip, causing the PMMA to reflow, or by removing the

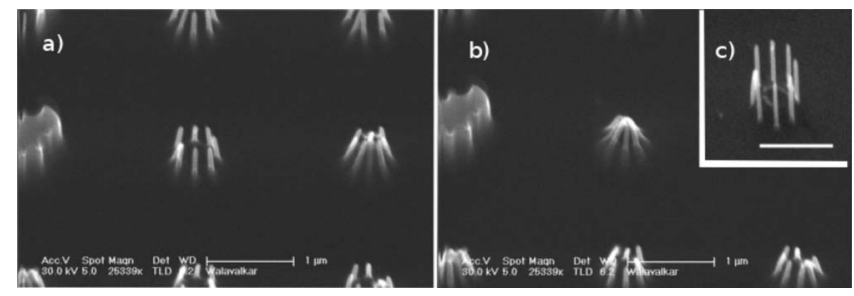

FIG. 4. (a) $500 \mathrm{~nm}$ wide circle of $50 \mathrm{~nm}$ diameter pillars, (b) same array after deformation, (c) same array after oxygen plasma release. Scale bar in picture is 1 micron. 

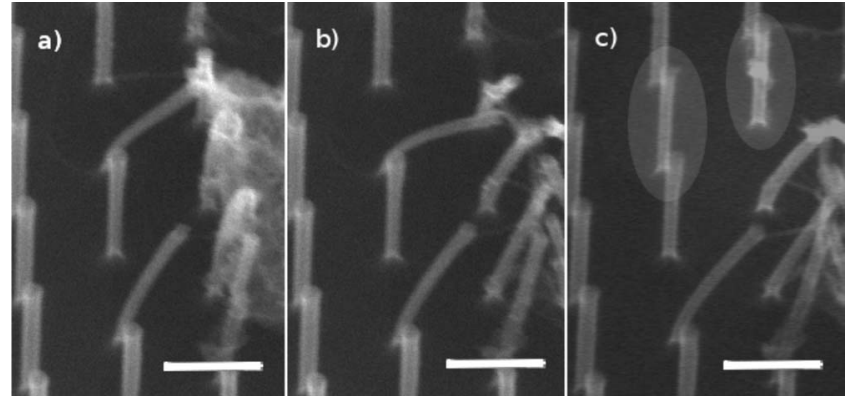

FIG. 5. (a) Clump of polymer stuck to top of pillars begins to bend the array, (b) same array after full deformation, (c) same array after polymer has been partially removed. Note the highlighted pillars have snapped back upright. Also note that the still bent pillars are held in place by strands of polymer. Scale bars are $250 \mathrm{~nm}$.

PMMA with an oxygen plasma (inset, Fig. 4). After a plasma clean it was possible to repeat the process of straining and unstraining the pillars. This cyclic loading without fracture has been previously demonstrated in amorphous silicon samples. ${ }^{27}$

For illustration of the extent of possible bending a segment of polymer was attached to the entirety of a set of pillars and contracted with an electron beam [Fig. 5(a)]. Figure 5(b) shows the near-horizontal bending of the pillar under full contraction. Finally, Fig. 5(c) highlights two pillars that have returned to their original configuration after partial polymer removal via electron beam ablation. It is important to note that the pillars still held in their bent configuration in frame $\mathrm{c}$ are held in place by still present strands of polymer.

\section{RESULTS AND DISCUSSION}

An application of this procedure is the ability to predictably incorporate enormous amounts of strain into nanostructures. In order to estimate the strain induced in the pillars, we analyze the structure in a manner similar to Timoshenko's treatment of a bimetallic strip. ${ }^{28}$ The actual geometry and material characteristics of the crosslinked region are difficult to measure and likely nonuniform, in reality behaving as a distributed film rather than an isolated region with clear boundaries. Additionally, the system could be complicated by slip at the interface, nonlinear elastic behavior of the polymer, and increasingly surface-dominated mechanical characteristics at the nanoscale. In spite of the simplified model, however, the proposed treatment shows excellent agreement with experimental data. Furthermore, the analysis predicts a constant radius-of-curvature, yielding a conservative estimate of the maximum strain.

We model the pillar as a cylinder with diameter $d$, and approximate the crosslinked region of PMMA which contributes to the deformation as a semicircular shell around the pillar with radial thickness $t$ and originally spun to a film thickness $L$ [Fig. 6(a)]. After exposure, an equivalent, freestanding crosslinked region would undergo a uniform vertical contraction by an amount $\Delta L$, corresponding to a unit contraction $\alpha \equiv \Delta L / L$. The length mismatch between the submerged portion of the pillar and the contracted PMMA causes the pillar to bend to an angle $\theta$ relative to the original pillar axis and substrate normal.
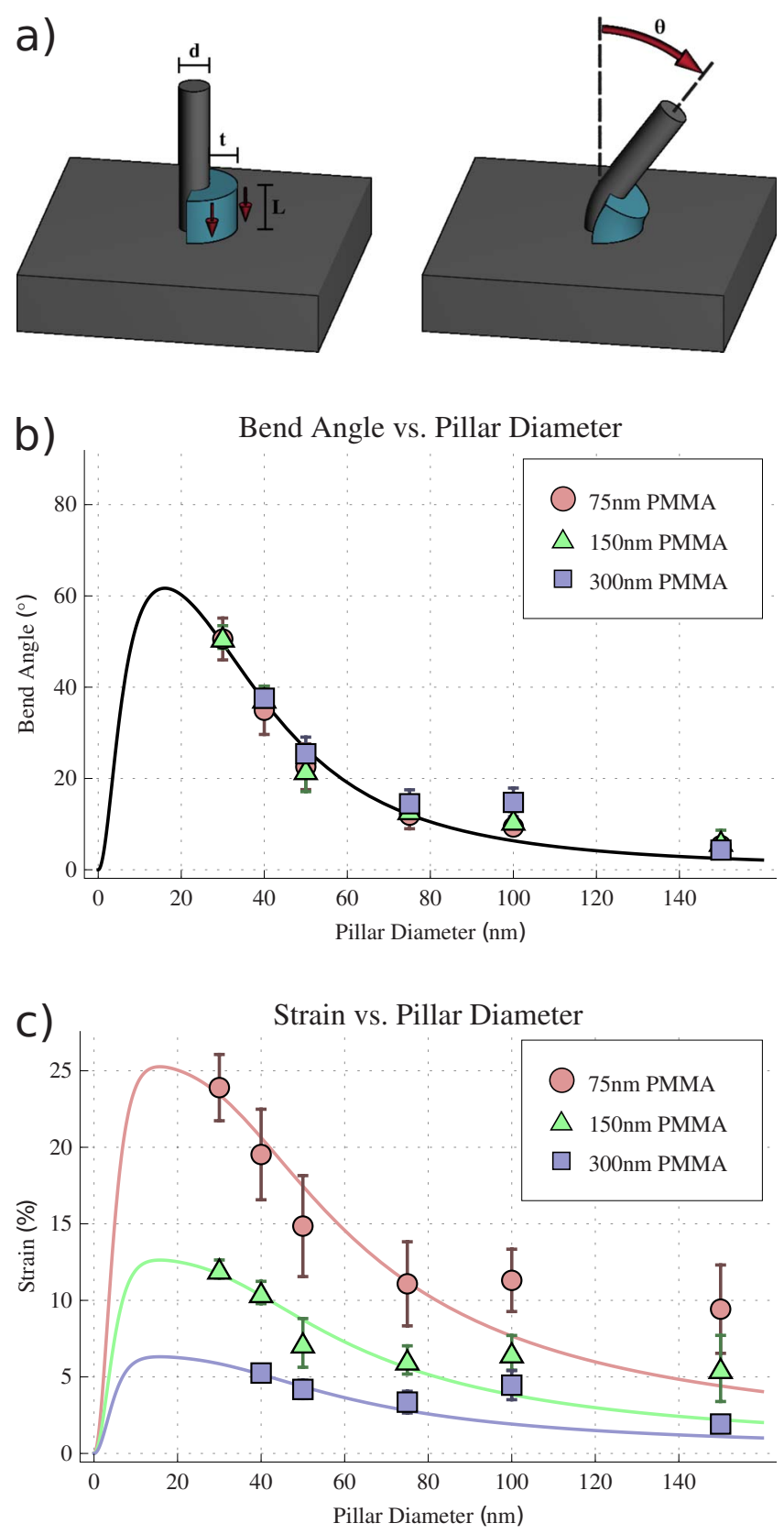

FIG. 6. (Color online) (a) Diagram illustrating the theoretical model. A small, semicircular region of PMMA adjacent to a silicon pillar undergoes vertical contraction due to electron-beam-induced crosslinking, yielding large pillar deformation. In the experiment, the crosslinked region is controlled by asymmetric electron-beam exposure, and is embedded in a continuous polymer film. (b) Variation in the maximum deformation angle with the diameter of the pillars, for various resist thicknesses. Theoretical model is independent of resist thickness. (c) Variation in the estimated strain in the pillars with the diameter of the pillars, for varying resist thicknesses. Points represent averages over several measurements, with error bars indicating the standard deviations.

Following Timoshenko, we find:

$$
\frac{1}{\rho}=\frac{\alpha\left(C_{p}-C_{s}\right)}{\frac{I_{s}}{A_{s}}+\frac{I_{p}}{A_{p}}+\frac{E_{s} I_{s}}{E_{p} A_{p}}+\frac{E_{p} I_{p}}{E_{s} A_{s}}+\left(C_{p}-C_{s}\right)^{2}},
$$

where $\rho$ is the radius of curvature, $\alpha \equiv \Delta L / L$ is the unit contraction of the PMMA, $C_{p}, C_{s}$ denote the center-of-mass coordinates, $I_{p}, I_{s}$ the cross-sectional moments, $A_{p}, A_{s}$ the areas, 
and $E_{p}, E_{s}$ the Young's moduli for the PMMA and silicon, respectively. The arclength along the neutral axis remains at the original polymer thickness $L$, yielding an exit angle $\theta$ $=L / \rho$

$$
\theta=\frac{L \alpha\left(C_{p}-C_{s}\right)}{\frac{I_{s}}{A_{s}}+\frac{I_{p}}{A_{p}}+\frac{E_{s} I_{s}}{E_{p} A_{p}}+\frac{E_{p} I_{p}}{E_{s} A_{s}}+\left(C_{p}-C_{s}\right)^{2}} .
$$

For the geometry described above and a coordinate system where $x=0$ corresponds to the center of the pillar, we have

$$
\begin{aligned}
& C_{s}=0 \quad C_{p}=\frac{3 d^{2}+6 d t+4 t^{2}}{3 \pi(d+t)}, \\
& A_{s}=\frac{\pi d^{2}}{4} \quad A_{p}=\frac{\pi t(d+t)}{2}, \\
& I_{s}=\frac{\pi d^{4}}{64} \quad I_{p}=A_{p}\left(\frac{d^{2}+2 d t+2 t^{2}}{8}-C_{p}^{2}\right) .
\end{aligned}
$$

Taking $E_{s}=160 \mathrm{GPa}$ and $E_{p} \approx 5 \mathrm{GPa}$ as the Young's modulus for the overexposed PMMA ${ }^{26}$ we solve for the remaining free parameters using a least-squares fit between the measured pillar angles and our analytic expression for $\theta$, obtaining $\Delta L \approx 43 \mathrm{~nm}$ and $t \approx 46 \mathrm{~nm}$. This contraction is on the order of the vertical contraction in overexposed PMMA reported elsewhere. ${ }^{26}$ While not measured directly, we estimate the total load applied to the pillars to be on the order of $10 \mu \mathrm{N}$ by finite element simulations.

The results are shown in Fig. 6(b), which illustrates close agreement between the experimental and analytical bend angles. It should be noted that although the wires are approximately a micron in length the entire strained region is concentrated within the PMMA layer resulting in a large observed bend angle as the pillar leaves the PMMA. Interestingly, the bend angle data does not show any measurable dependence on resist thickness. In the analytic expression, this corresponds to a fixed $\Delta L$ rather than a fixed unit contraction $(\alpha)$ as one might expect. The exact cause of this behavior is unclear and requires further study, but might indicate that the deformation is occurring over a length which is smaller than the total film thickness. If this is the case, the strain required to accomplish the same deformation over a reduced distance would be higher than that estimated below. Were this distance also fixed, the strain curves would be independent of the PMMA starting thickness.

To provide a conservative strain estimate, we assume for now that the deformation is uniform and occurs over the entire submerged length of the pillar; we note that the portion of the pillar which extends beyond the PMMA remains unstrained. Using $\epsilon \equiv \Delta \rho / \rho$ for the strain, we find the maximum strain along the edge of the pillar

$$
\epsilon=\frac{\Delta L\left(C_{p}-C_{s}\right)\left(C_{n}-C_{e}\right)}{L\left[\frac{I_{s}}{A_{s}}+\frac{I_{p}}{A_{p}}+\frac{E_{s} I_{s}}{E_{p} A_{p}}+\frac{E_{p} I_{p}}{E_{s} A_{s}}+\left(C_{p}-C_{s}\right)^{2}\right]},
$$

where $\Delta \rho=C_{n}-C_{e}$ is the distance between the neutral axis $\left(C_{n}\right)$ and the far edge of the pillar $\left(C_{e}\right)$

$$
C_{n}=\frac{E_{s} A_{s} C_{s}+E_{p} A_{p} C_{p}}{E_{s} A_{s}+E_{p} A_{p}} \quad C_{e}=-\frac{d}{2} .
$$

The results are plotted in Fig. 6(c), and demonstrate that we can controllably incorporate $23.9 \%$ strain in $30 \mathrm{~nm}$ diameter single crystal silicon nanowires. The strain profile within the pillar is anisotropic; based on the location of the neutral axis, it is possible to introduce both tensile and compressive strain or solely tensile. For large pillar diameters, the neutral axis lies inside the pillar, resulting in tensile strain at the outer edge and compressive strain on the inner. As the diameter decreases, however, the neutral axis moves toward the PMMA. For $C_{n}>d / 2$, corresponding to $d \leqslant 17.6 \mathrm{~nm}$, the strain is tensile throughout the pillar cross section.

It is important to recall that the stress is only applied within the 75-300 nm of PMMA, resulting in high values of strain but only in the submerged region of the pillar. Furthermore, these values fall within the range of reported values for strain in silicon nanowires under fracture-free polymerization incited deformation. ${ }^{21,22}$ Possible mechanisms that could allow such extreme behavior have been studied previously $^{14-16}$ and rely on the relatively small volumes of nanowires resulting in the fabrication of statistically "defectfree" structures that lack sites for fracture nucleation. Additionally, the small diameter of the nanowires could possibly allow for dislocations to be "annealed" out of a structure by diffusing to the perimeter. ${ }^{21}$

Unlike these studies, ${ }^{21,22}$ since our deformation only occurred within the PMMA layer while the uncovered region remained unaffected, it was impossible to perform an in situ HRTEM on the strained region to atomistically quantify the deformation as either elastic or plastic. However, to SEM resolution each pillar appeared to undergo a macroscopically elastic deformation, returning to its original position after being freed from the PMMA even after several tens of bending and unbending cycles.

Anisotropic strain has recently been exploited as a method for breaking the inversion symmetry in silicon photonics, ${ }^{12,13}$ introducing a second order nonlinearity. Furthermore, such asymmetrically strained materials can exhibit interesting optical selection rules ${ }^{11}$ based on the strained splitting of the degenerate light and heavy hole bands. Previously proposed methods to introduce strain typically rely on the deposition of lattice mismatched layers, a method which can incorporate only a few percent of strain, and which is fixed at fabrication time. ${ }^{10,11}$ In contrast, the method present in this work allows for the incorporation of several tens of percent of strain as well as being fully tunable and reversible.

Another application presented in this paper is the ability to monitor the manipulation of pillars in real time via SEM while capturing an object. Here we used an array of pillars that had been selectively actuated to capture a $300 \mathrm{~nm}$ aluminum oxide polishing bead [Fig. 7(a)]. During capture, the contraction of the pillars squeezed a collection of beads such that they were forced through the top of the closed pillars [Fig. 7(b)]. Once the pillars had been closed, the resist was selectively removed and the captured collection of beads remained trapped within the pillars. We expect this technique 


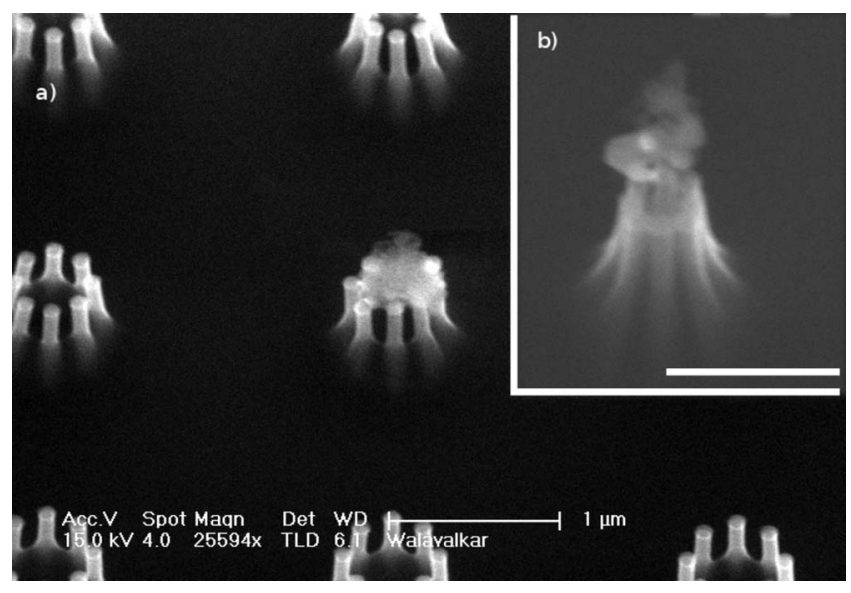

FIG. 7. (a) $300 \mathrm{~nm}$ alumina polishing bead caught by $100 \mathrm{~nm}$ pillars. (b) 75 $\mathrm{nm}$ pillars catching a collection of smaller alumina polishing beads. The scale bar is $500 \mathrm{~nm}$.

to have important biological applications such as capturing individual cells for further study. Compared to electrically actuated nanotweezers, ${ }^{8}$ the polymer-controlled nanowires may be more densely packed, allowing the study of interactions between neighboring traps, and offer the ability to leave the tweezers closed without any external input.

\section{CONCLUSION}

This work demonstrates the use of PMMA as a force mediating layer which actuates silicon nanowires. Using standard top-down fabrication to define the pillars, PMMA was spun on and planarized to make the force mediating layer. By overexposure in an SEM, selected regions of the PMMA were crosslinked to actuate the pillars. It was further demonstrated that this could be reversed by heating or by removing the PMMA with an oxygen plasma. The deformation remained fixed after exposure to ambient atmosphere due to the stability of the overexposed PMMA, and excess PMMA was stripped to allow further fabrication. Using arrays of nanowires, we trapped alumina spheres, confirming that the flexible nature of the pillars can be utilized for capturing nanometer scale objects. In addition, this technique yields reversible strain of $24 \%$ in silicon nanowires, which may potentially exhibit interesting optical and electrical phenomena.

\section{ACKNOWLEDGMENTS}

The authors gratefully acknowledge support from the Defense Advanced Research Projects Agency (DARPA) under the NACHOS program, Award No. W911NF-07-1-0277, the University of Arizona and the National Science Founda- tion under the CIAN program, sponsor number Y502628, prime Award No. EEC-0812072, and the Kavli Nanoscience Institute. Andrew Homyk thankfully recognizes support from the ARCS foundation. David Henry appreciates support from the John and Fannie Hertz foundation, and we thank Adrian Chapman for useful discussions. Sameer S. Walavalkar and Andrew P. Homyk contributed equally to this work.

${ }^{1}$ A. R. Guichard, D. N. Barsic, S. Sharma, T. I. Kamins, and M. L. Brongersma, Nano Lett. 6, 2140 (2006).

${ }^{2}$ L. Sainiemi, H. Keskinen, M. Aromaa, L. Luosujarvi, K. Grigoras, T. Kotiaho, J. Makela, and S. Franssila, Nanotechnology 18, 505303 (2007).

${ }^{3}$ D. Scheible and R. Blick, Appl. Phys. Lett. 84, 4632 (2004).

${ }^{4}$ H. Kim, M. Qin, H. Westphall, L. Smith, and R. Blick, Nanotechnology 18, 4 (2007).

${ }^{5}$ L. Lauhon, M. Gudiksen, D. Wang, and C. Lieber, Nature (London) 420, 57 (2002).

${ }^{6}$ A. Nassiopoulos, S. Grigoropoulos, and D. Papadimitriou, Appl. Phys. Lett. 69, 2267 (1996).

${ }^{7}$ P. Photopoulos, A. Nassiopoulou, D. Kouvatsos, and A. Travlos, Appl. Phys. Lett. 76, 3588 (2000).

${ }^{8}$ P. Bøggild, T. Hansen, C. Tanasa, and F. Grey, Nanotechnology 12, 331 (2001).

${ }^{9}$ Z. Tang, Y. Xu, G. Li, and N. Aluru, J. Appl. Phys. 97, 114304 (2005).

${ }^{10} \mathrm{~J}$. Singh, Electronic and Optoelectronic Properties of Semiconductor Structures (Cambridge University Press, Cambridge, 2003), Chap. 1.4, pp. 26-31.

${ }^{11}$ P. Hashemi, L. Gomez, J. Hoyt, M. Robertson, and M. Canonico, Appl. Phys. Lett. 91, 083109 (2007).

${ }^{12}$ R. Jacobsen, K. Andersen, P. Borel, J. Pedersen, L. Frandsen, O. Hansen, M. Kristensen, A. Lavirnenko, G. Moulin, H. Ou, C. Peucheret, B. Zsigri, and A. Bjarkalev, Nature (London) 441, 199 (2006).

${ }^{13}$ N. Hon, K. Tsia, D. Solli, and B. Jalali, Appl. Phys. Lett. 94, 091116 (2009).

${ }^{14}$ B. Wu, A. Heidelberg, and J. Boland, Nature Mater. 4, 525 (2005).

${ }^{15}$ J. Zhang, Y. Zhao, and B. Palosz, Appl. Phys. Lett. 90, 043112 (2007).

${ }^{16}$ M.-F. Yu, B. S. Files, S. Arepalli, and R. S. Ruoff, Phys. Rev. Lett. 84, $5552(2000)$.

${ }^{17}$ E. Wong, P. Sheehan, and C. Lieber, Science 277, 1971 (1997).

${ }^{18}$ X. Han, Y. F. Zhang, K. Zheng, X. N. Zhang, Z. Zhang, Y. J. Hao, X. Y. Guo, J. Yuan, and Z. L. Wang, Nano Lett. 7, 452 (2007).

${ }^{19}$ J. R. Greer, J.-Y. Kim, and M. J. Burek, Journal of Materials 61, 19 (2009).

${ }^{20}$ C.-L. Hsin, W. Mai, Y. Gu, Y. Gao, C.-T. Huang, Y. Liu, L.-J. Chen, and Z.-L. Wang, Adv. Mater. 20, 3919 (2008).

${ }^{21}$ K. Zheng, X. Han, L. Wang, Y. Zhang, Y. Yue, Y. Qin, X. Zhang, and Z. Zhang, Nano Lett. 9, 2471 (2009).

${ }^{22}$ X. Han, K. Zheng, Y. Zhang, X. Zhang, Z. Zhang, and Z. L. Wang, Adv. Mater. 19, 2112 (2007).

${ }^{23}$ O. Lourie, D. M. Cox, and H. D. Wagner, Phys. Rev. Lett. 81, 1638 (1998).

${ }^{24}$ M. Henry, S. Walavalkar, A. Homyk, and A. Scherer, Nanotechnology 20, 255305 (2009).

${ }^{25}$ I. Zailer, J. Frost, V. Chabasseur-Molyneux, C. Ford, and M. Pepper, Semicond. Sci. Technol. 11, 1235 (1996).

${ }^{26}$ W. H. Teh, C.-T. Liang, M. Graham, and C. G. Smith, J. Micro/Nanolith. MEMS MOEMS 12, 641 (2003).

${ }^{27}$ C. Gaire, D.-X. Ye, T.-M. Lu, G.-C. Wang, and R. C. Picu, J. Mater. Res. 23, 328 (2008).

${ }^{28}$ S. Timoshenko, J. Opt. Soc. Am. 11, 233 (1925). 